\title{
Writeback-Aware LLC Management for PCM-Based Main Memory Systems
}

\author{
BAHAREH POURSHIRAZI, University of Illinois at Chicago \\ MAJED VALAD BEIGI, Northwestern University \\ ZHICHUN ZHU, University of Illinois at Chicago \\ GOKHAN MEMIK, Northwestern University
}

\begin{abstract}
With the increase in the number of data-intensive applications on today's workloads, DRAM-based main memories are struggling to satisfy the growing data demand capacity. Phase Change Memory (PCM) is a type of non-volatile memory technology that has been explored as a promising alternative for DRAM-based main memories due to its better scalability and lower leakage energy. Despite its many advantages, PCM also has shortcomings such as long write latency, high write energy consumption, and limited write endurance, which are all related to the write operations.

In this article, we propose a novel writeback-aware Last Level Cache (LLC) management scheme named WALL to reduce the number of LLC writebacks and consequently improve performance, energy efficiency, and lifetime of a PCM-based main memory system. First, we investigate the writeback behavior of LLC sets and show that writebacks are not uniformly distributed among sets; some sets observe much higher writeback rates than others. We then propose a writeback-aware set-balancing mechanism, which employs the underutilized LLC sets with few writebacks as an auxiliary storage for the evicted dirty lines from sets with frequent writebacks. We also propose a simple and effective writeback-aware replacement policy to avoid the eviction of the dirty blocks that are highly reused after being evicted from the cache.

Our experimental results show that WALL achieves an average of $30.9 \%$ reduction in the total number of LLC writebacks, compared to the baseline scheme, which uses the LRU replacement policy. As a result, WALL can reduce the memory energy consumption by $23.1 \%$ and enhance PCM lifetime by $1.29 \times$, on average, on an 8-core system with a 4GB PCM main memory, running memory-intensive applications.
\end{abstract}

CCS Concepts: $\bullet$ Computer systems organization $\rightarrow$ Processors and memory architectures; $\bullet$ Hardware $\rightarrow$ Emerging technologies;

Additional Key Words and Phrases: Phase change memory, write endurance, energy consumption, performance, last level cache

This work is an extension of our previous work, which is published on Proceedings of the Design, Automation, and Test in Europe 2018 (DATE'18) with the title "WALL: A Writeback-Aware LLC Management for PCM-based Main Memory Systems."

This work is supported in part by the National Science Foundation under Grant No. CCF-1513899.

Authors' addresses: B. Pourshirazi and Z. Zhu, Electrical and Computer Engineering Department, University of Illinois at Chicago, Chicago, IL 60607, USA; emails: \{bpours2, zzhu\}@uic.edu; M. Valad Beigi, Electrical Engineering and Computer Science Department, Northwestern University, Evanston, IL 60208, USA; email: majed.beigi@northwestern.edu; G. Memik, Electrical Engineering and Computer Science Department, Northwestern University, Evanston, IL 60208, USA; email: memik@eecs.northwestern.edu.

Permission to make digital or hard copies of all or part of this work for personal or classroom use is granted without fee provided that copies are not made or distributed for profit or commercial advantage and that copies bear this notice and the full citation on the first page. Copyrights for components of this work owned by others than ACM must be honored. Abstracting with credit is permitted. To copy otherwise, or republish, to post on servers or to redistribute to lists, requires prior specific permission and/or a fee. Request permissions from permissions@acm.org.

(C) 2019 Association for Computing Machinery.

1084-4309/2019/01-ART18 \$15.00

https://doi.org/10.1145/3292009

ACM Transactions on Design Automation of Electronic Systems, Vol. 24, No. 2, Article 18. Pub. date: January 2019. 


\section{ACM Reference format:}

Bahareh Pourshirazi, Majed Valad Beigi, Zhichun Zhu, and Gokhan Memik. 2019. Writeback-Aware LLC Management for PCM-Based Main Memory Systems. ACM Trans. Des. Autom. Electron. Syst. 24, 2, Article 18 (January 2019), 19 pages.

https://doi.org/10.1145/3292009

\section{INTRODUCTION}

DRAM can no longer satisfy the memory capacity demands of the modern-day applications due to its scalability limit; it is difficult to scale DRAM cells down to feature sizes smaller than $16 \mathrm{~nm}$ [34]. Moreover, DRAM consumes considerable amount of static and refresh power. It is estimated that DRAM power constitutes $20 \%$ to $40 \%$ of the total system's energy in today servers [16]. Phase Change Memory (PCM) is a promising technology that is gaining interest as a DRAM replacement for building future main memories $[2,21,20,23,26]$. PCM is a random-access memory that is about 2 to 4 times denser than DRAM [14]. In addition to its scalability, zero standby power, low read latencies, and resiliency to soft errors are some of the intriguing features of a PCM-based main memory [26]. However, PCM suffers from some major shortcomings including long write latency, high write energy consumption, and limited write endurance, which are all related to write operations. The impact of write operations on PCM will be discussed in detail in Section 2.2.

To deal with the above-mentioned overheads of write operations in PCM, there are two common types of solutions. First, category is the optimizations on the PCM architecture to minimize the impact of writes. For example, modifying the request scheduling policy in the PCM main memory to alleviate the performance overheads of writes on reads [1,24, 32, 38]; or modifying the PCM main memory architecture to reduce or balance the write loads on PCM cells and enhance their lifetime [37]. Second, category is reducing the total number of writes sent to the PCM main memory by modifying the Last Level Cache (LLC)'s management policies [28]. Our work falls into the latter category.

We propose WALL, a novel dynamic writeback-aware LLC management scheme to improve performance, energy efficiency, and lifetime of a PCM-based main memory system by reducing the number of writebacks from LLC to PCM. In general, WALL consists of a writeback-aware set balancing mechanism and a writeback-aware replacement policy. Writebacks of the last level cache are not uniformly distributed among its sets; some sets have far more writebacks than others, while some sets rarely see writebacks (please see Section 3.1 for detailed results). The proposed set balancing mechanism reduces the number of writebacks by employing the underutilized sets with infrequent writebacks as auxiliary storage units (inside LLC) for the evicted dirty lines from sets with many writebacks. Moreover, the proposed writeback-aware replacement policy tries to keep the dirty blocks that are frequently accessed after eviction in LLC. To do so, it allows the dirty eviction victims (i.e., dirty LRU block) to stay in the cache and be re-accessed; if the block becomes LRU block again without being accessed, then it will be evicted from LLC then.

To implement the set balancing mechanism of WALL, we first proposed a simple partner assignment strategy [22]. The simple partner assignment strategy classifies sets into three categories: (1) writer, or sets with frequent writebacks; (2) non-writer, or sets with infrequent writebacks; and (3) neutral, sets that are neither writer nor non-writer. Each writer set is partnered with a non-writer set until no non-writer sets are left un-partnered. Although this partner assignment strategy has simple implementation and works effectively for many workloads, it also has two limitations. First, it cannot always balance the number of writer and non-writer sets; thereby some writer or non-writer sets may remain without partners. Second, it does not guarantee the inclusion of writer sets with the largest number of writebacks or non-writer sets with the smallest number 


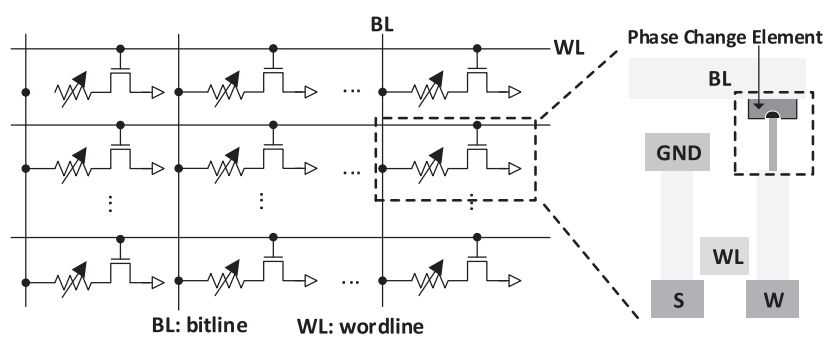

Fig. 1. A schematic showing how PCM cells can be addressed, and a close-up view of the phase-change element [11].

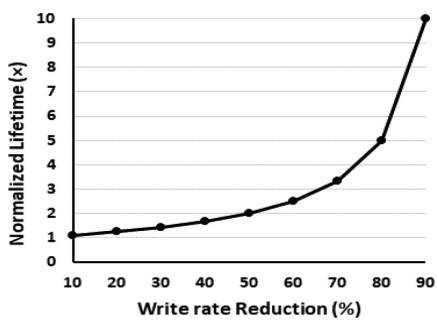

Fig. 2. The impact of reducing write traffic on PCM lifetime.

of writebacks in the partner assignment process. We further propose three novel partner assignment strategies called contraction, expansion and ConExp to alleviate the limitations of the simple partner assignment strategy. Specifically, when the number of writer and non-writer sets are imbalanced, the expansion strategy includes some of the neutral sets, which are the most eligible to be considered writer or non-writer, in the partner assignment process. However, the contraction strategy addresses the second limitation of the simple partner assignment strategy by first assigning partners to writer sets with the largest number of writebacks and non-writer sets with the smallest number of writebacks. The ConExp strategy is a combination of the two contraction and expansion strategies to deal with both limitations of the simple partner assignment strategy.

We evaluate our proposed schemes by running SPEC CPU2006, NAS and PARSEC workloads on gem5 [10] integrated with modified NVMAIN [19], which simulates the PCM-based main memory system. The experimental results indicate that our scheme can reduce the total number of LLC writebacks by $30.9 \%$, on average, compared to a baseline scheme, which uses the LRU replacement policy. As a result, for a system with eight cores and a 4GB PCM main memory, it can enhance PCM lifetime by $1.29 \times$, on average, and reduce the memory energy consumption by $23.1 \%$, on average.

The rest of this article is organized as follows. Section 2 discusses background. Section 3 describes the proposed scheme. Section 4 presents the experimental results. The related work is discussed in Section 5. Finally, Section 6 summarizes the work.

\section{BACKGROUND}

\subsection{Phase Change Memory (PCM)}

Phase Change Memory (PCM) is a type of non-volatile memory technology that has been extensively explored as a DRAM alternative. Figure 1 illustrates the structure of a PCM cell. To store bit information, a PCM cell uses the phase change property of a material known as chalcogenide that can be in two physical states: amorphous state that has high resistance and represents bit "0" and crystalline state that has low resistance and represents bit "1" [26, 30]. Writing on a PCM cell is performed by heating the storage material via applying a short but high voltage pulse and then cooling it down. The rate at which the material is cooled down determines the stored bit; slow cooling stores bit " 1 " and fast cooling stores bit " 0 " on the cell. To later read a PCM cell, the state of the material is detected by applying a low voltage to it [33]. The resistive characteristic of PCM cells allows them to retain their data for a long time with no need for periodic refreshes.

\subsection{Impact of Write Operations On PCM}

The main advantages of PCM over DRAM are its scalability and non-volatility. However, PCM has higher write access energy and latency than DRAM (2 to 8 times [14]) and limited write endurance. 

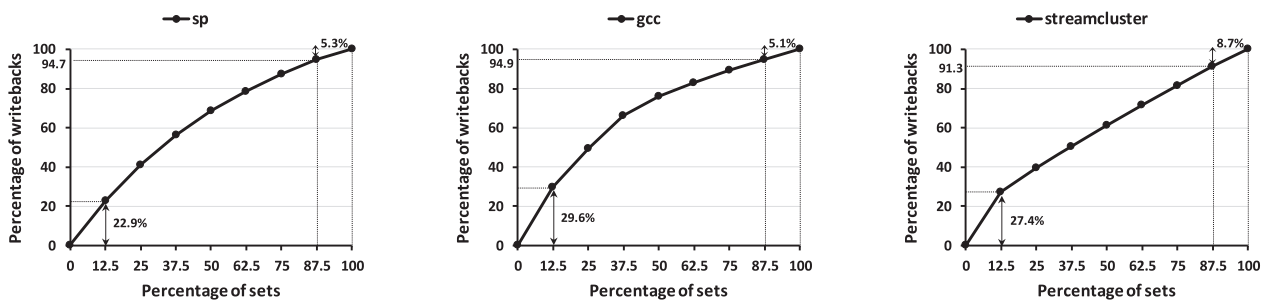

Fig. 3. Cumulative distribution of writebacks over LLC sets. (NOTE: sets are sorted in a descending order based on their total number of writebacks.)

A previous study [1] has shown that the long-latency write operations can increase the effective latency of read requests by 1.2 to 1.8 times. In addition, the number of write operations to PCM affects its lifetime. The PCM-based memory system lifetime can be estimated as [23]:

$$
\text { System Lifetime }=Y(\text { years }) \approx \frac{w_{\max } \cdot S}{B} \cdot 2^{-25}
$$

In this formula, $\boldsymbol{B}$ is the write traffic (or write rate, GBps), $\boldsymbol{Y}$ is the maximum number of years that a PCM with size $\boldsymbol{S}$ and cell endurance of $w_{\max }$ can last. The impact of reducing the write traffic on the expected PCM lifetime is shown in Figure 2. It should be noted that the results of the figure are independent of $S$ and $w_{\max }$. We also assume that the writes are distributed uniformly across the entire PCM memory.

\section{WALL SCHEME}

Our proposed scheme, WALL [22], consists of a writeback-aware set balancing mechanism and a writeback-aware replacement policy. The WALL set balancing mechanism classifies LLC sets into three categories, writers (i.e., frequent writeback sets), non-writers (i.e., infrequent writeback sets), and neutral. To reduce the number of write requests to PCM, the non-writer sets are used to store the evicted dirty lines from the writer sets. Specifically, each writer set is partnered with a non-writer set (until there is no non-writer set left) and upon eviction of a LRU dirty line from the writer set, the line will be inserted into the set's partner instead of being written back to the main memory. Besides, our writeback-aware replacement policy further reduces the number of LLC writebacks by keeping the frequent writeback blocks in the cache.

\subsection{Motivation}

In this section, we explain the motivation of our work by investigating LLC sets' writeback behavior. To this end, we have run three workloads, selected from different benchmark suites ( $s p$ from NAS [18], gcc from SPEC CPU2006, and streamcluster from PARSEC [4]) on our simulated system (please see Section 4.1 for more details). Figure 3 shows the cumulative distribution of writebacks over LLC sets.

The results reveal that majority of the writebacks from the LLC to main memory are generated by less than half of the LLC sets. For sp, $25 \%$ of the LLC sets, with the largest number of writebacks (i.e., frequent writeback sets), are responsible for $41.1 \%$ of the total number of writebacks. However, $12.5 \%$ of the sets, with the smallest number of writebacks (i.e., infrequent writeback sets), are accountable for only $5.3 \%$ of the total number of writebacks. In general, some sets tend to write back much more frequently than others. For example, since $22.9 \%$ of the writebacks for $s p$ are performed by $12.5 \%$ of the frequent writeback sets, a frequent writeback set writes to main memory about four times more frequently than an infrequent writeback set, on average. Similarly, for gcc, $25 \%$ of the frequent writeback sets perform $49.6 \%$ of the writebacks while the same percentage of the 
infrequent writeback sets are responsible for only $10.8 \%$ of the total number of writebacks. Finally, for streamcluster, $20.7 \%$ of the writebacks are sourced from $6.3 \%$ of the frequent writeback sets.

Based on this observation, we propose a set balancing mechanism to reduce the number of writes to a PCM-based main memory system. More specifically, WALL aims to prevent a noticeable percentage of write traffic from reaching the PCM main memory by taking the non-uniform distribution of LLC set writebacks into account. The idea of avoiding the eviction of LLC sets' highly reused dirty blocks, which are likely to become eviction victims soon after being re-inserted in the cache (i.e., frequent writeback blocks), has been discussed in a recent study called WADE [28]. WADE partitions the blocks of each LLC set into two groups, frequent writeback blocks and nonfrequent writeback blocks and tries to keep the frequent writeback blocks in the set to reduce the number of writes to the main memory. However, one major shortcoming of WADE is that for every set, irrespective of its characteristics, it considers the nonfrequent writeback blocks of the set as the only replacement candidates; while other sets might have clean, underutilized lines. Moreover, WADE is rather complex. In our study, a simple but effective writeback-aware replacement policy is also proposed to keep the frequent writeback blocks in the cache. Overall, WALL can reduce the number of LLC writebacks by considering the writeback behavior of sets and their blocks at the same time.

\subsection{Writeback-Aware Set Balancing Scheme}

To decide whether a set is a writer or non-writer, WALL monitors the number of writebacks and accesses of each LLC set for a time window. Generally, a set is "writer" if the number of writebacks from it exceeds a certain threshold (i.e., $\tau_{h i g h_{-} w b}$ ), but a set with relatively small number of writebacks is not necessarily a good partner set. A non-writer set must have enough space to store the evicted dirty blocks of its writer partner without noticeable performance penalty. To measure the degree to which a set can hold its working set, we employ a saturating arithmetic miss counter (i.e., saturation counter) like that in a previous study [27]. For a K-way set associative cache, the working range of the saturation miss counter is from 0 to $2 \mathrm{~K}-1$ [27]. Upon every access, the saturation counter is incremented if the access results in a miss, and it is decremented otherwise. To count the number of writebacks, we also use a saturating writeback frequency counter for each set that is incremented upon every writeback from the set. At the end of a monitoring period, we divide the writeback values by two (i.e., shift the counters right one bit). This reduces the impact of set's writeback behavior in the past on the type of the set in the current time period.

WALL considers a set "non-writer" if both of saturation and writeback counters are smaller than specific thresholds (i.e., $\tau_{\text {sat }}, \tau_{l o w \_} w$ ). For a set with writeback frequency counter of $W$ and saturation counter of $M$, the set is considered writer if $\left(W \geq \tau_{\text {high_wb }}\right)$, non-writer if $\left(M \leq \tau_{\text {sat }}\right.$ \& $\left.W \leq \tau_{l o w_{-} w b}\right)$ and neutral otherwise. We divide the total execution time of programs into epochs of $10^{7}$ accesses to the LLC. On entering an epoch, the partnership between two sets can be easily broken if the writer set has no blocks in its non-writer partner. The thresholds are re-calculated and if there is a pair of writer and non-writer sets with no partners, they will be assigned to each other. Note that for a set with partner (i.e., a writer set with blocks in its partner or a non-writer set that holds some of its partners' blocks), we do not change the set type. Our experiments show that set types rarely change after an initial set type identification epoch and most partnerships remain intact. However, to avoid overfill a non-writer set, if the saturation counter of a non-writer set reaches $\alpha \times \tau_{\text {sat }}$, where $\alpha$ is 2 in our experiments, then the insertion of blocks from its writer partner will be suspended until the set's saturation counter retrieves to values smaller than $\tau_{\text {sat }}$. To reduce implementation complexities, we assume each writer set is partnered with only one nonwriter set. In other words, the number of the paired writer and non-writer sets is equal to the size 


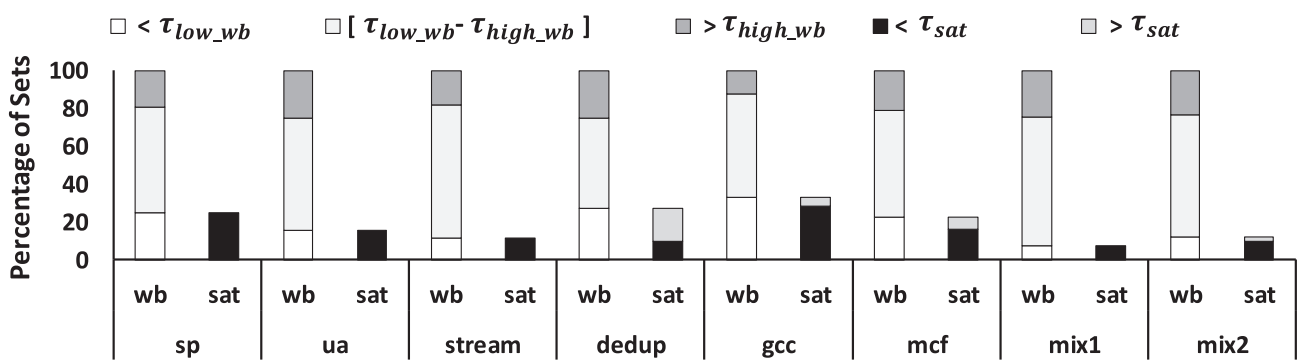

Fig. 4. Distribution of LLC sets based on the thresholds (wb: writebacks; sat: saturation; NOTE: sat bars are only for the sets with $\left.w b<\tau_{l o w_{-} w b}\right)$.

of the smaller group of writer and non-writer sets. We later discuss how accesses to sets and their partners are handled in Section 3.4.

To determine the writeback thresholds, three simple steps are followed. First, the arithmetic mean of all the writeback values is computed and referred to as the overall average. Then, $\tau_{l o w_{-} w b}$ is computed as the arithmetic mean of the writeback values smaller than the overall average. Finally, $\tau_{\text {high }} w b$ is computed as the arithmetic mean of the writeback values larger than the overall average. Note that since these thresholds cannot guarantee an equal number of writer and non-writer sets, some writer sets may remain without a partner at the end. Moreover, we assume $\tau_{\text {sat }}=\mathrm{K} / 4$ (i.e., $\mathrm{K}$ is the set associativity). Figure 4 depicts the distribution of sets based on the discussed thresholds for the workloads shown in Table 3 (see Section 4.1). The results are based on the values obtained after the initial epoch of $10^{7}$ accesses to the LLC. It should be noted that the saturation bars (i.e., bars with horizontal axis title of sat) in the figure are only for the sets with writeback counters smaller than $\tau_{l o w_{-} w b}$. Our results show that the selected thresholds can distinguish different types of sets from each other effectively.

\subsection{Writeback-Aware Replacement Policy}

In addition to assigning partners to the writer sets, WALL enables a writeback-aware replacement policy inside the LLC sets to further reduce the number of writebacks to the PCM. To keep the frequent writeback blocks in the cache, such blocks need to be identified first. We propose a much simpler, yet effective method compared to the prediction scheme discussed in WADE [28], because such prediction schemes are usually complex and costly in terms of area, energy, and/or performance overheads.

The intrinsic definition of a frequent writeback block is a block that is frequently reused each time after being evicted from the cache. Generally, our scheme avoids the eviction of such blocks by giving the dirty victims a second chance to stay in the cache and be accessed again. To keep track of the dirty blocks that have been given a second chance, a one-bit flag called $F V$ (i.e., Former Victim) is considered for each block. We assume the baseline replacement policy is LRU. When a replacement is needed in a LLC set, the dirty status bit of the LRU line (i.e., the eviction victim) is checked; if the LRU block is clean, then it will be evicted from the cache, but if the block is dirty, then two scenarios are possible. First, the block is not a former victim $(F V=0)$. In this case, the line will be moved to the MRU position of the access stack and will be marked as a former victim (i.e., its $F V$ flag will be set to "1"), this process will be repeated until finding an eviction victim. Second, the block is a former victim $(F V=1)$ and has become the eviction victim for the second time without being accessed. In this case, the block will be evicted from the cache. If a cache line with $F V=1$ is accessed, then its $F V$ bit will be reset to "0." The reason is that such block is likely 


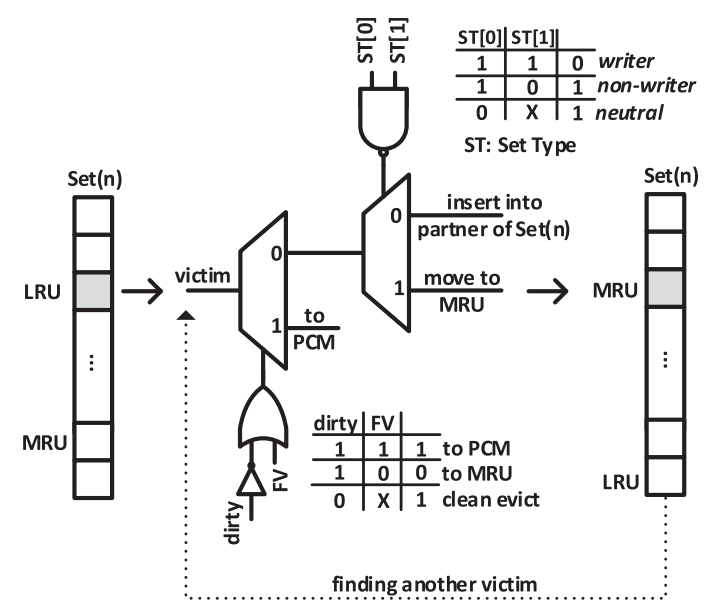

Fig. 5. WALL design.

to be a frequent writeback block. It should be noted that all these steps happen in parallel with the resolution of the miss, thereby there is no performance penalty.

We use the proposed replacement policy for the non-writer and neutral sets. Since writer sets usually have high miss rate values and often choose dirty blocks as eviction victims, for those sets, we use the baseline LRU replacement policy. However, on eviction of a LRU dirty block from a writer set with a partner, the block will be inserted into the set's partner.

\subsection{Set Balancing Simple Partner Assignment and Access Management}

For writer sets with large number of writebacks, changing the replacement policy may cause a nontrivial increase in the sets' miss rates. Hence, the writeback-aware replacement policy is applied to those sets that are not writer (i.e., neutral and non-writer sets). Instead, WALL virtually increases the associativity of a writer set by assigning a non-writer partner to it. We first propose a simple partner assignment strategy and access management for set-balancing LLC. The reason that we have excluded the neutral sets from the partnering process is that it is not beneficial to write from one set to another set with similar writeback or miss frequencies.

The partner of a writer set is selected randomly from the non-writer sets. To keep tracking the partners, a small direct-access remap table is introduced. The indices of the sets' partners are saved in the remap table, which is indexed by set indices. For a set with no partner, its own index is stored. When a LRU dirty line is evicted from a writer set with a partner, it will be inserted into the set's partner. To show whether a block in a non-writer set is repositioned from the set's writer partner or not, a one-bit flag called $\boldsymbol{R B}$ (i.e., Repositioned Block) is considered for each block. The remap table is also augmented with one-bit flag $\boldsymbol{P}$ (i.e., Partnered) for each set to show whether a writer set has any blocks in its partner or not. Upon an access to a writer set, if the access results in a miss, then the remap table is checked; if $P$ is " 1 ," then the set's partner will be accessed for the block; otherwise, main memory will be accessed as usual. If the access also misses in the set's partner, then main memory must be accessed. If all the repositioned blocks of a writer set get evicted from its partner, then $P$ flag of the writer set will be reset to "0."

The design of WALL is depicted in Figure 5. On an eviction of a block from a LLC set, we first decide whether the block needs to be written back to the PCM or not. If not, then depending on the set type, the block either remains in the set as MRU or will be written back into the set's partner. 


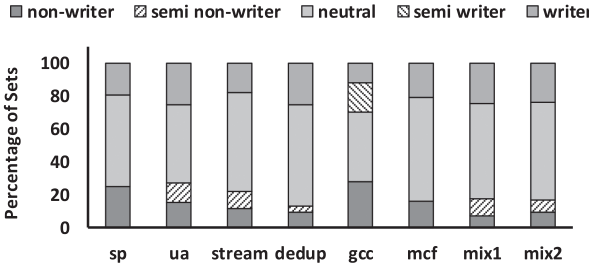

Fig. 6. Distribution of LLC sets after applying the expansion strategy.

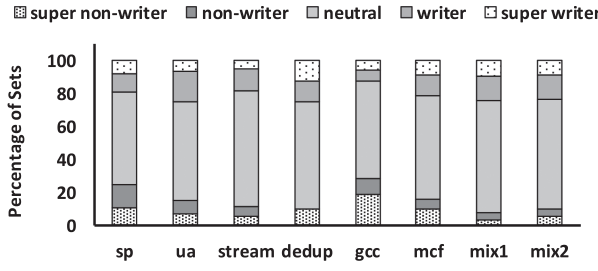

Fig. 7. Distribution of LLC sets after applying the contraction strategy.

To specify the type of each set, a 2-bit register called ST is considered per set (i.e., the set is writer if ST = "11," non-writer if ST = "10," and neutral if ST = " $00 "$ or " 01 ").

\subsection{Extended Partner Assignment Strategies}

The simple partner assignment strategy of WALL (explained in Section 3.4) cannot guarantee an equal number of writer and non-writer sets. In other words, some writer sets may remain without partners at the end of the partner assignment process. In this section, to efficiently exploit the available storage space of the non-writer sets and to further reduce the number of writebacks from the writer sets, we propose three more partner assignment strategies called expansion, contraction and ConExp, which is a combination of contraction and expansion strategies.

3.5.1 Expansion Partner Assignment Strategy. The expansion strategy modifies the writeback thresholds to balance the number of writer and non-writer sets. Specifically, when the number of non-writer sets is considerably smaller than the writer sets, the expansion strategy finds the most eligible neutral sets to be added to the non-writer sets. However, when the number of writer sets is smaller, the expansion strategy assigns the remaining (without partners) non-writer sets, to the neutral sets that can benefit the most from the partnership.

To avoid enlarging the group of writer or non-writer sets unreasonably (i.e., causing more imbalance in the sizes of the two groups), we define an expansion condition; the expansion strategy is applicable only when the size of the larger group is at least $\beta \times$ the size of the smaller group. Our evaluations show that $\beta=1.5$ provides the best balance between the number of writer and non-writer sets. The writeback thresholds of the expansion strategy are represented as $\tau_{\text {exp } \_l o w \_w b}$ and $\tau_{\exp \_h i g h \_w b}$. When the expansion condition is true, considering a neutral set with writeback frequency counter of $W$ and saturation counter of $M$, the two possible scenarios are as follows: (1) If non-writer sets are fewer, then the neutral set is considered semi non-writer if $M \leq \tau_{\text {sat }}$ and $W \geq \tau_{\exp \_h i g h \_w b}$. To determine $\tau_{\exp \_l o w_{\_} w b}$, the arithmetic mean of the writeback values between $\tau_{l o w_{-} w b}$ and the overall average is computed. (2) If writer sets are fewer, then the neutral set is considered semi writer if $W \leq \tau_{\exp \_l o w_{-} w b} . \tau_{\exp \_h i g h \_w b}$ is also computed as the arithmetic mean of the writeback values between the $\tau_{h i g h \_w b}$ and the overall average. Figure 6 shows the distribution of sets with the expansion strategy after the initial monitoring epoch.

The expansion strategy starts by assigning the original writer and non-writer sets as partners. Then, it continues by assigning the remaining writer/non-writer sets to the semi non-writer/semi writer sets until no semi non-writer/non-writer set remains without a partner.

3.5.2 Contraction Partner Assignment Strategy. Since the number of writer and non-writer sets are usually not the same, some sets remain without a partner. However, it is beneficial to always keep the writer sets with the largest number of writebacks, or "super writer sets," and nonwriter sets with the smallest number of writebacks, or "super non-writer" sets, included in the partner assignment process. The contraction strategy distinguishes the super writer and super 
Table 1. Total Storage Overhead

\begin{tabular}{|l|l||l|l|}
\hline Type & Storage & \multicolumn{1}{|c|}{ Type } & Storage \\
\hline$F V$ per block & $16 \mathrm{~KB}$ & Saturation counter (6-bit) per set & $3 \mathrm{~KB}$ \\
\hline$R B$ per block & $16 \mathrm{~KB}$ & Writeback counter (8-bit) per set & $4 \mathrm{~KB}$ \\
\hline$P$ per set & $0.5 \mathrm{~KB}$ & Remap table & $6 \mathrm{~KB}$ \\
\hline$S T$ per set & $1 \mathrm{~KB}$ & TOTAL & $\mathbf{4 6 . 5 \mathrm { KB }}$ \\
\hline
\end{tabular}

non-writer sets from the rest of the sets using two writeback thresholds called $\tau_{c o n \_h i g h \_w b}$ and

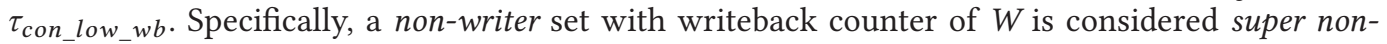
writer if $W \leq \tau_{\text {con_low_wb }}$ and a writer set with writeback counter of $W$ is considered super writer if $W \geq \tau_{c o n \_h i g h \_w b}$. To determine the $\tau_{c o n_{-} l o w_{-} w b}$, the arithmetic mean of the writeback values smaller than the $\tau_{l o w_{-} w b}$ is computed. Also, $\tau_{c o n_{-} h i g h_{-} w b}$ is computed as the arithmetic mean of the writeback values larger than the $\tau_{h i g h} w b$. Figure 7 illustrates the distribution of sets with the contraction strategy after the initial monitoring epoch.

The contraction strategy starts from assigning partners to super writer and super non-writer sets and then continues by assigning partners to the remaining writer and non-writer sets.

3.5.3 Contraction-Expansion (ConExp) Strategy. There are two main shortcomings with the expansion strategy. First, since the semi non-writer sets are less effective than the non-writer sets in reducing the number of writebacks, assigning them as partners to the super writer sets should be avoided. Second, although the expansion strategy tries to balance the number of writer and non-writer sets, the super writer or super non-writer sets may still be excluded from the partner assignment process. To alleviate these problems, we propose the ConExp strategy, which is a combination of the contraction and expansion strategies. ConExp assigns partners, in order, to the super sets, then to the original writer and non-writer sets, and finally to the semi sets, until no non-writer or semi non-writer set remains without a partner.

It is worth mentioning that the semi and super writer sets are represented (treated the same) as the writer sets and the semi and super non-writer sets as the non-writer sets. Hence, these strategies do not require any additional hardware overheads.

\subsection{Overhead Analysis}

Table 1 summarizes the storage overhead of WALL. The total storage overhead of WALL is less than $0.6 \%$ of the LLC capacity. It is worth mentioning that this overhead is about half of that of WADE [28].

Calculating the writeback thresholds and updating the remap table (i.e., pairing writer and nonwriter sets) are only performed once at the end of each epoch. Hence, their performance impacts are negligible.

\section{RESULTS}

\subsection{Methodology}

In this work, we model an 8-core processor using the gem5 full-system simulator integrated with NVMAIN. The system configuration of our experiments is shown in Table 2. The PCM configurations are generated by NVSIM [9] and CACTI [5], the cell parameters used in NVSIM are based on the projections by Reference [7]. The benchmarks used in this study are chosen from NAS [18], SPEC CPU2006, and PARSEC [4] as depicted in Table 3. The selected benchmarks are some of the memory-intensive workloads from the three benchmark suites. For all the workloads, we use either 
Table 2. System Configuration

\begin{tabular}{|l|l|}
\hline \multicolumn{2}{|c|}{ Processor and On-chip Caches } \\
\hline Cores & 8 cores, out-of-order, 2.0 $\mathrm{GHz}$ \\
\hline L1-I/D & Split 32KB I/D-cache/core, 4-way, 8-MSHR, 2-cycle hit \\
\hline L2 & 256KB/core, 8-way, 12-MSHR, 12-cycle hit \\
\hline L3 (LLC) & SRAM: Shared, 8MB, 32-way, 32-MSHR, 35-cycle hit \\
\hline Coherency & MOESI directory, 2 $\times 4$ grid packet NoC, XY routing \\
\hline \multicolumn{2}{|c|}{ Main Memory } \\
\hline PCM & $\begin{array}{l}\text { 4GB, 4 Channel, 1 rank/channel, 4 banks/rank, 400MHz } \\
t_{S E T}=150 \mathrm{~ns}, t_{R E S E T}=100 \mathrm{~ns}, t_{R C D}=120 \mathrm{~ns} \\
\text { Cell endurance }=32 \times 10^{6} \text { writes }\end{array}$ \\
\hline MC & $\begin{array}{l}\text { Four controllers, Open page, 32-entry queues (one read and one write } \\
\text { queue), Write drain threshold: high }=80 \%, \text { low }=50 \%, \text { Address mapping: } \\
\text { page interleaving }\end{array}$ \\
\hline
\end{tabular}

Table 3. Evaluated Workloads Characteristics

\begin{tabular}{|lcc||lcl|}
\hline Workload & RPKI & WPKI & Workload & RPKI & WPKI \\
\hline \hline \multicolumn{5}{|c|}{ from the NAS benchmarks (8-Thread) } \\
\hline \hline $\mathrm{sp}$ & 4.98 & 2.55 & ua & 3.12 & 2.67 \\
\hline \hline \multicolumn{5}{|c|}{ from the PARSEC benchmarks (8-Thread) } \\
\hline \hline stream & 24.4 & 0.21 & dedup & 11.5 & 8.32 \\
\hline \hline \multicolumn{5}{|c|}{ from the SPEC CPU2006 benchmarks } \\
\hline \hline $8 \times$ gcc & 7.42 & 1.59 & $8 \times$ mcf & 43.5 & 9.02 \\
\hline mix1 & 2.91 & 1.90 & mix2 & 12.7 & 4.21 \\
\hline mix1: $4 \times$ lbm, $4 \times$ bzip & & mix2: $4 \times$ cactusADM, $4 \times$ leslie3D \\
\hline
\end{tabular}

RPKI/WPKI: main memory reads/writes per 1,000 instructions.

sampled reference or native input sets to represent a real-world execution scenario and run the applications for two billion instructions, after two billion instructions for cache warm-up phase. In this article, we compare WALL with (1) Baseline that uses the LRU replacement policy, (2) Baseline double-way, a baseline cache of the same size with double the associativity, and (3) WADE, which is the scheme proposed in Reference [28]. In PCM, we always prioritize reads over writes if write queue is less than $80 \%$ full.

\subsection{LLC Writeback Reduction}

Figure 8 shows the writeback reduction of WALL for the different types of LLC sets. The results are normalized to the baseline scheme. It is worth mentioning that any writeback of a writer set's lines from its partner is considered for the original writer set.

WALL when using the simple partner assignment strategy achieves an average of $26.6 \%$ writeback reduction, compared to the Baseline scheme. The three main reasons for this reduction are: (1) The efficacy of the set type identification process. (2) The capability of the writeback-aware set balancing scheme in reducing the writer sets' writebacks; the number of writebacks originated from writer sets is reduced by $39.5 \%$, on average (i.e., from $33.4 \%$ to $20.1 \%$ of the Baseline total writebacks), while the writebacks originated from non-writer sets are increased slightly from $10.4 \%$ to $13.1 \%$ of the Baseline total number of writebacks. (3) The proposed writeback-aware replacement 


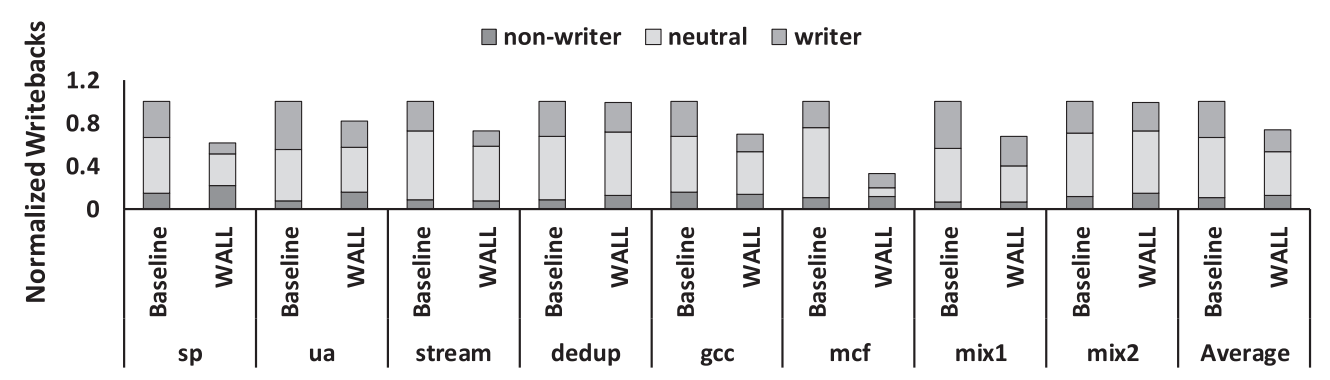

Fig. 8. WALL's normalized LLC writebacks reduction.

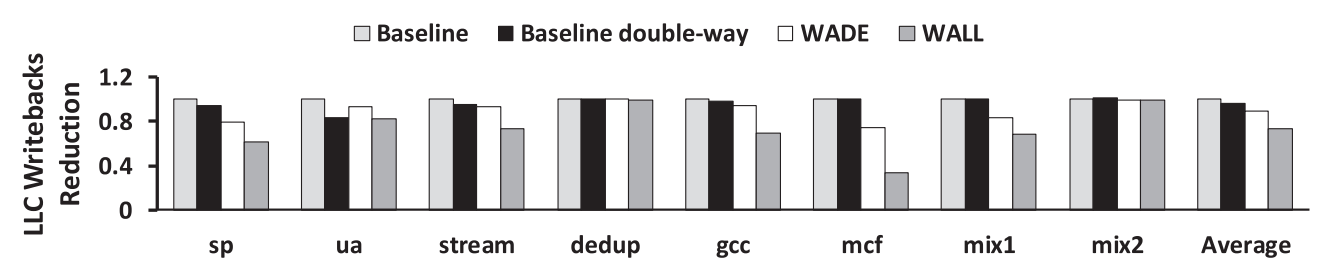

Fig. 9. Writebacks reduction of evaluated schemes.

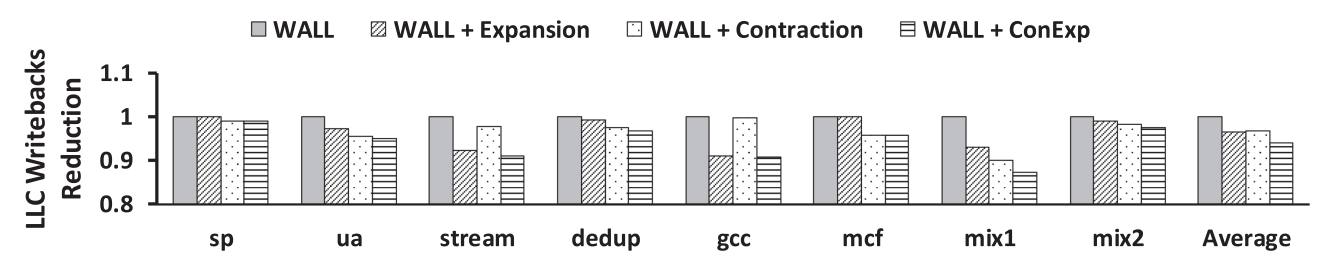

Fig. 10. Writebacks reduction of WALL with different partner assignment strategies.

policy has been able to reduce the writebacks originated from the neutral sets by $28.6 \%$, on average (i.e., from $56.2 \%$ to $40.1 \%$ of the Baseline total writebacks). It is worth noting that for some of the benchmarks, the proposed replacement policy has also been able to reduce the number of writebacks originated from the non-writer sets. Figure 9 compares the normalized writebacks reduction of the evaluated schemes. Compared with Baseline double-way and WADE, WALL reduces the number of writebacks by $23.3 \%$ and $16.4 \%$, on average, respectively. Based on the results, duplicating the set associativity is not very helpful in reducing the number of LLC writebacks; our baseline implementation is 32-way and increasing the associativity beyond that does not cause a significant improvement.

Figure 10 compares the writeback reduction of the extended partner assignment strategies proposed in Section 3.5. The results are normalized to the simple partner assignment strategy. The expansion strategy can reduce the number of writebacks by $3.6 \%$, on average (by up to $9.2 \%$ for gcc). This reduction is caused by including more writer (or semi writer) sets in the partner assignment process. The contraction strategy can reduce the number of writebacks by $3.4 \%$, on average (by up to $4.6 \%$ for $u a$ ). The reason for this reduction is the fact that sets with highest write frequency (super writer sets) are always assigned a partner, which is in the super non-writer sets (i.e., one that has the smallest write frequency). ConExp, which uses the benefits of both strategies, can reduce the number of writebacks by $5.9 \%$, on average, compared to the simple partner assignment strategy. In other words, ConExp strategy can reduce the number of writebacks by $30.9 \%$ compared to the Baseline scheme. 


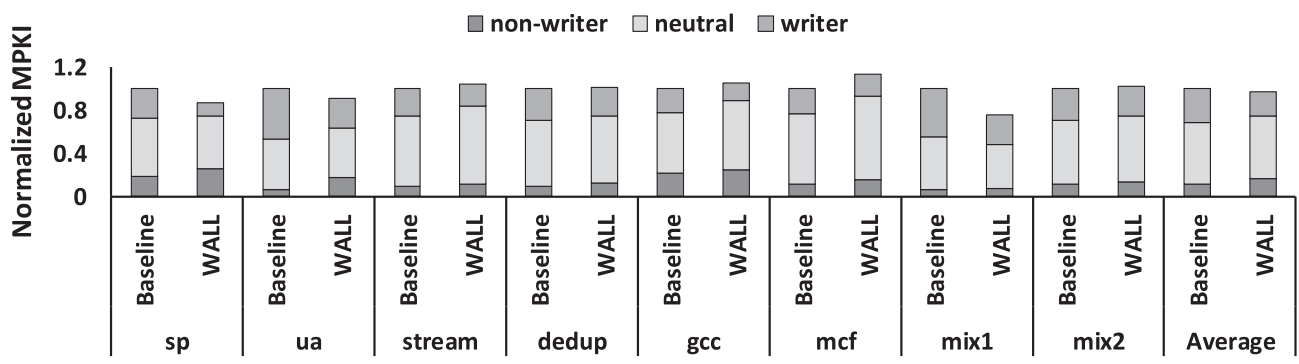

Fig. 11. WALL's normalized MPKI.

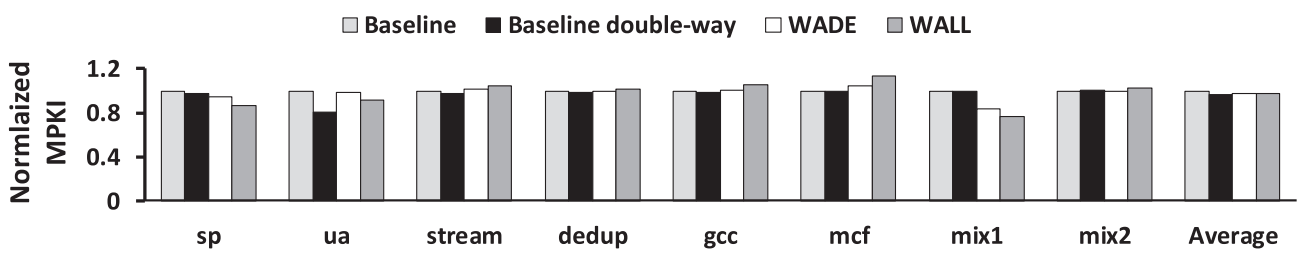

Fig. 12. MPKI of evaluated schemes.

\subsection{LLC Miss Rate}

Figure 11 shows the normalized MPKI (Misses Per Kilo Instructions) of WALL for different types of LLC sets. The accesses that result in a hit in a writer set's partner are considered for the original writer set. Figure 12 shows the normalized MPKI of the evaluated schemes.

Results reveal that WALL when using the simple partner assignment strategy reduces the MPKI by $2.4 \%$, on average, compared to the Baseline. This reduction is mainly because WALL stores the frequently reused dirty blocks of writer sets in the non-writer partners and performs similarly to doubling the associativity of those cache sets. More specifically, the MPKI of writer sets is reduced by $27.8 \%$, on average (i.e., from $30.7 \%$ to $22.1 \%$ of the Baseline total MPKI). However, the MPKI is increased from $12.0 \%$ to $16.2 \%$ for the non-writer sets and from $57.3 \%$ to $59.1 \%$ of the Baseline total MPKI for the neutral sets, on average, respectively. For some cases, the writeback-aware replacement policy has incurred miss penalties by evicting the clean lines that are later reused more frequently than the saved dirty blocks (i.e., those that have been given a second chance). For other cases, the dirty lines kept in LLC by the writeback-aware replacement policy are re-referenced more than the lines evicted by them instead. It should be noted that the penalty is small, because we give only a second chance to the dirty victims of the sets. The set-balancing scheme reduces the MPKI of writer sets at the cost of increasing the MPKI of non-writer sets. However, since the nonwriter sets are usually underutilized, the increase is small. Compared with the LRU replacement policy (used for both Baseline and Baseline double-way), our scheme has higher management cost and increases the MPKI for some benchmarks. However, our evaluations reveal that it does not have a noticeable negative impact on the cache miss rate. Compared with Baseline double-way, WALL increases the MPKI by $1.0 \%$, on average. But WALL reduces the MPKI by $0.3 \%$, on average, compared to $W A D E$, because $W A D E$ does not distinguish different set types.

\subsection{Energy Comparison}

Figure 13 shows the normalized energy of the PCM main memory for the evaluated schemes. WALL when using the simple partner assignment strategy can save main memory's energy by $19.2 \%$, on average, compared to the Baseline. Compared with Baseline double-way and WADE, WALL reduces 


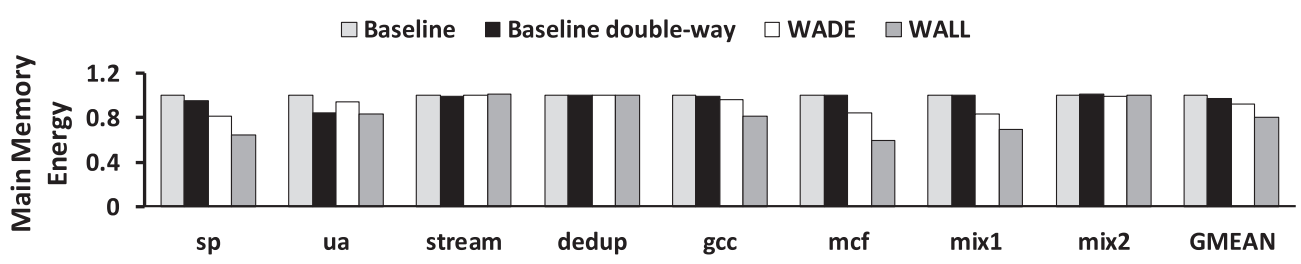

Fig. 13. Normalized main memory energy.

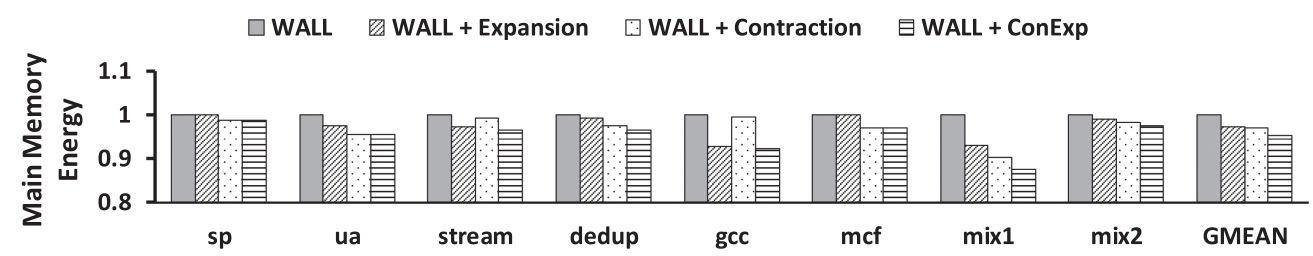

Fig. 14. Main memory energy of WALL with different partner assignment strategies.

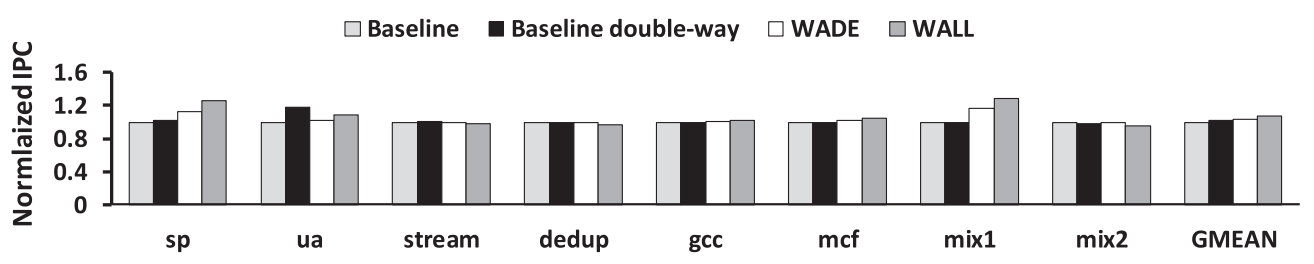

Fig. 15. Normalized IPC.

the energy consumption of the PCM main memory by $16.5 \%$ and $11.3 \%$, on average, respectively. The energy consumption of writing to PCM is much higher than that of reads. Hence, the energy saving is mainly due to the reduction in the number of write requests issued to PCM by WALL. That is also the reason that Baseline double-way exhibits a higher power consumption compared to WALL; Baseline double-way experiences a smaller MPKI, but a higher rate of writebacks. As discussed in Section 3.1, unlike WADE, which uses complex prediction schemes, the energy consumption of the logic components added to LLC by WALL is negligible.

Figure 14 shows the normalized energy of the main memory for the extended partner assignment strategies. Compared to the simple partner assignment strategy, the expansion and the contraction strategies can save main memory's energy consumption by $2.7 \%$ and $3.0 \%$, on average, respectively. ConExp can reduce the main memory's energy by $4.8 \%$, on average, compared to the simple partner assignment strategy. In other words, ConExp strategy can reduce the energy consumption of the PCM main memory by $23.1 \%$, on average, compared to the Baseline scheme.

\subsection{Performance Comparison}

Figure 15 compares the normalized system IPC of the evaluated schemes. The overhead of the second searches in partners of the writer sets is considered in our experiments.

WALL when using the simple partner assignment strategy improves performance by $6.7 \%$, on average, compared to the Baseline scheme. Compared with Baseline double-way and WADE, WALL improves system performance by $4.9 \%$ and $3.2 \%$, on average, respectively. The latency of writing to PCM is much higher than that of reads. Hence, the reduced average access latency of the PCM main memory is the major reason for the performance improvement. The results indicate that WALL can reduce the PCM average access latency by $12.6 \%$, on average, compared to the Baseline. 


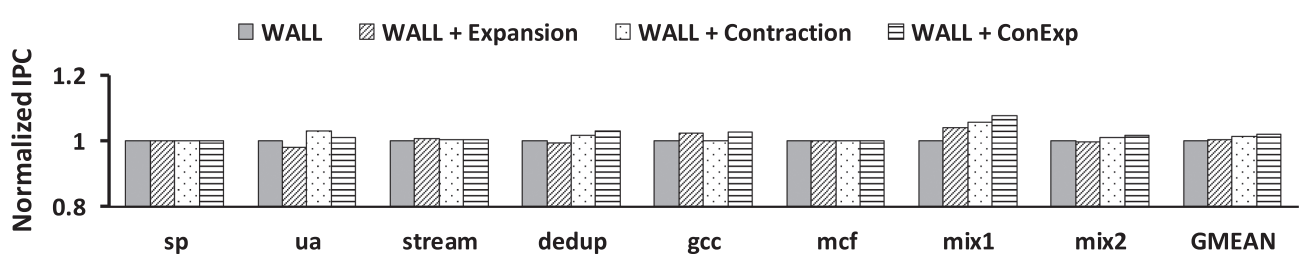

Fig. 16. Normalized IPC of WALL with different partner assignment strategies.

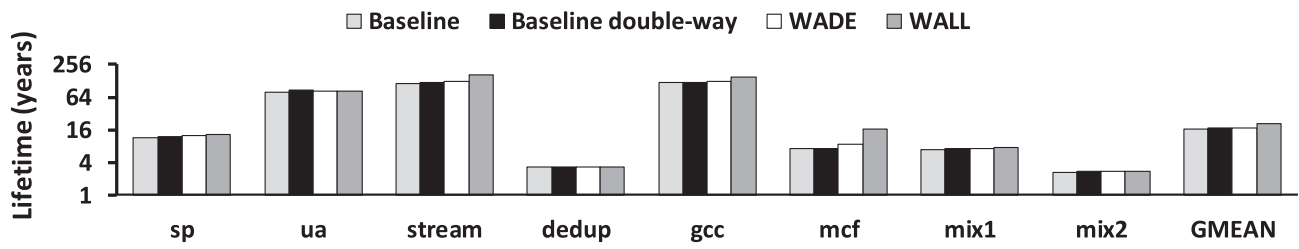

Fig. 17. Lifetime enhancement (years, log scale).

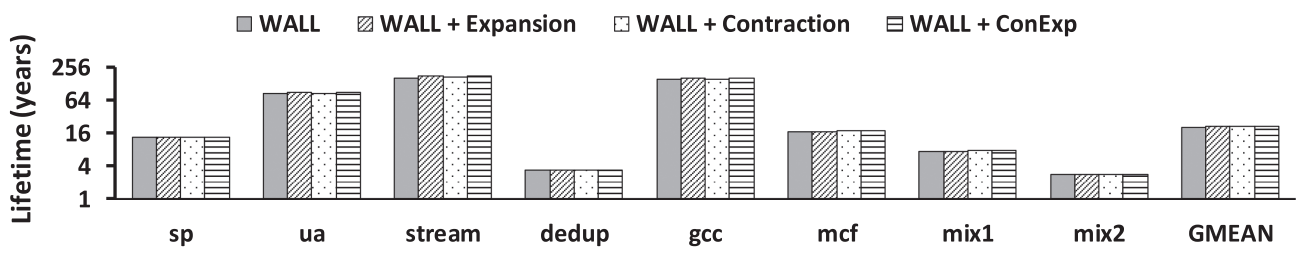

Fig. 18. Lifetime enhancement of WALL with different partner assignment strategies.

The reduction is due to reducing the PCM write traffic and thus the queuing delay of the PCM read requests.

Figure 16 illustrates the normalized system IPC of WALL with the extended partner assignment strategies. Compared to the simple partner assignment strategy, the expansion and contraction strategies can improve the system performance by $0.7 \%$ and $1.5 \%$, on average, respectively. ConExp, which uses the benefits of the both strategies, can improve performance by $2.1 \%$, on average, compared to the simple partner assignment strategy. Compared to Baseline, ConExp strategy can improve performance by $8.7 \%$, on average.

\subsection{PCM Lifetime Enhancement}

Figure 17 compares the PCM lifetime enhancement of the evaluated schemes. The lifetime is calculated based on the analytical model explained in Section 2.2. It should be noted that PCM lifetime is inversely proportional to the writes per cycle or write rate (GBps). WALL when using the simple partner assignment strategy can enhance PCM lifetime by $1.25 \times$, on average, compared to the Baseline. Compared with Baseline double-way and WADE, WALL enhances the PCM lifetime by $1.21 \times$ and $1.17 \times$, on average, respectively. Alleviating the write traffic sent out to the PCM main memory by WALL is the reason for the lifetime enhancement.

Figure 18 shows the PCM lifetime enhancement of the extended partner assignment strategies. ConExp can enhance PCM lifetime by $1.04 \times$, on average, compared to the simple partner assignment strategy. In other words, ConExp strategy can enhance PCM lifetime by $1.29 \times$ compared to the Baseline scheme. 


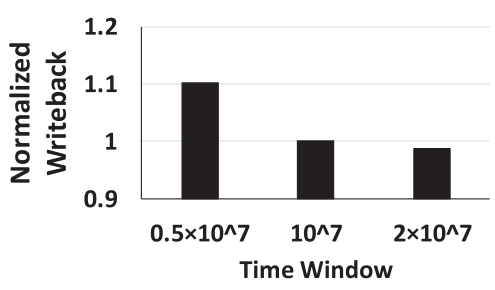

Fig. 19. Writeback reduction of WALL for three different time window sizes.

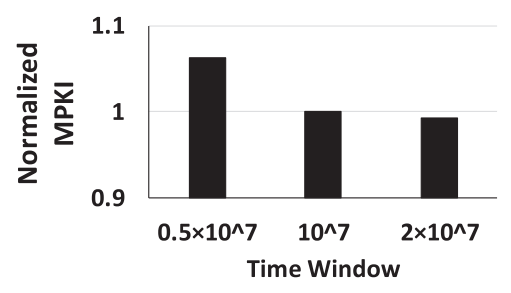

Fig. 20. MPKI of WALL for three different time window sizes.

\subsection{Impact of Time Window Size}

For experimental results presented so far, the set types are checked and adjusted with an epoch of $10^{7}$ LLC accesses, because our experiments indicate that this provides the best balance between the writeback reduction and storage overhead. Next, we discuss the impact of time window size. We choose to use the number of LLC accesses instead of the number of cycles, because WALL works based on the number of writebacks/accesses of the sets and epochs with the same number of cycles may have very different number of writebacks/accesses. In other words, to obtain a consistent approach among the epochs, we choose the number of LLC accesses rather than cycles.

Figures 19 and 20 compare the average writeback and miss rate values of WALL + ConExp for three different time window sizes. The results are normalized to $10^{7}$ LLC access time windows. Our goal is to reduce the number of writebacks while keeping the storage overhead at minimum. The evaluations show that using time windows of $0.5 \times 10^{7}$ accesses results in $10.3 \%$ more writebacks and $6.3 \%$ higher miss rate compared to time windows of $10^{7}$ accesses. The reason is that time windows of $0.5 \times 10^{7}$ accesses are not long enough, resulting in incorrect identification of some set types. Identifying a neutral set as non-writer and assigning a writer partner to it can increase its writeback and miss rate values, because the set will not have enough space for its writer partner's blocks. Moreover, identifying a writer set as neutral can also increase its writeback and miss rate values, because writer sets need to be managed differently, as we explained in Section 3.2. Finally, identifying a neutral set as writer or a non-writer set as neutral may prevent us from using the set-balancing scheme efficiently.

Our results also show that for time windows of $2 \times 10^{7}$ accesses, larger writeback counters are required; otherwise, counters will saturate, causing inaccurate identification of set types. Using larger writeback counters improves the accuracy of the set type identification. However, based on our evaluations, even with the higher storage overhead, the writeback reduction is small (only $1.2 \%$ ) compared to $10^{7}$ access time windows. So, overall, the selected time windows of $10^{7}$ accesses to the LLC gives us the best balance between the writeback reduction and storage overhead. It is also worth mentioning that there is no benefit in using even larger time window sizes (e.g., $10^{8}$ accesses), because not only the storage overhead would be much higher but also any required updates on the set types would be postponed to later times in the program execution, which in turn can reduce the benefits of WALL.

\section{RELATED WORK}

Many recent studies have focused on mitigating the overheads of write operations in PCM-based main memories. Lee et al. [15] proposed a scheme called eager writeback, which writes the LRU dirty cache lines back into the main memory before their eviction to improve system performance. A variation of eager writeback is proposed in Reference [25] to improve PCM performance by early and eagerly writing back the long latency SET operations. In Reference [24], the concept of write cancellation is proposed to prioritize reads over writes; to immediately service the incoming reads, 
this scheme cancels the conflict writes. The performance overhead of write operations is alleviated by parallelizing read or write accesses with an ongoing write in Reference [1]. Moreover, Zhou et al. [38] developed a non-blocking PCM bank design that aims to service subsequent reads or writes in parallel with an on-going write. Xia et al. [32] explored the possibility of removing the unmodified data from a single write and then, merging modified data of multiple writes to be sent within one write request to improve PCM write bandwidth. In addition, Zhang et al. [37] has shown that only a small portion of the main memory is frequently accessed in a given time period. Based on that observation, their scheme records and predicts the memory regions' write frequencies to select a proper write latency (i.e., the number of SET iterations) for every incoming memory write operation to improve system performance and memory lifetime. In this work, we focus on reducing the number of write requests issued to PCM. Since our design only reduces the number of writebacks from the last-level cache and does not change the main memory's architecture, it can be used along with other techniques that optimize the PCM architecture to minimize the impact of writes.

There have also been other studies proposed to reduce the write overheads of other types of non-volatile memory technologies such as STT-RAM and ReRAM. Zhang et al. [36] proposed a scheme called Mellow Writes that extends memory controllers to selectively perform slow writes to reduce the impact of writes on endurance and performance of the ReRAM-based memories. In Reference [3], a temperature aware scheme is proposed to enhance the lifetime and performance of ReRAM-based memories by reducing the number of write accesses to the hot ReRAM banks. Kultursay et al. [13], investigated the possibility of replacing DRAM with STT-RAM for main memories. In that work, STT-RAM write overheads are reduced by bypassing the row buffer writes and tracking dirty blocks to perform partial writes within a row. STT-RAM is typically considered as an alternative to SRAM caches or used in SRAM-NVM hybrid caches. To alleviate the write overheads of STT-RAM in a hybrid cache, Wang et al. [29] presented an adaptive placement and migration policy based on the access pattern of different classes of write operations in LLC. Wu et al. [31] partitioned hybrid cache into read and write regions and migrated cache blocks within the cache to mitigate the write overheads of STT-RAM. In this work, we have focused on PCM as one of the most extensively studied alternatives for DRAM-based main memories due to its high density and low cost. However, WALL can also be used for other types of non-volatile main memory technologies (e.g. ReRAM) with similar shortcomings related to the write operations.

Some studies have proposed techniques for reducing the number of LLC writebacks to the nonvolatile component of a hybrid main memory consisting of NVM and DRAM [6,35]. In Reference [6], a miss penalty-aware LRU-based cache replacement policy, called MALRU is proposed to consider the asymmetry of cache miss penalty on DRAM and NVM. MALRU keeps the high-latency NVM blocks as well as the low-latency DRAM blocks with good temporal locality in a reserved area to protect them from being evicted. Similarly, Zhang et al. [35] proposed a write-back aware LLC management scheme for the hybrid main memory systems to reduce the number of writebacks to NVM by improving the hit ratio of the NVM memory blocks in the cache. However, those techniques are only applicable for the hybrid main memories.

The set balancing cache (i.e., $S B C$ ) proposed in Reference [27] tries to balance the pressure on cache sets by associating sets with maximum saturation counters with sets with small saturation counters. Though SBC reduces cache miss rate, it is not always able to reduce the number of writebacks. Our experiments show that, not every set with a maximum saturation counter has large number of writebacks (e.g., a set may evict clean lines mostly). However, there are some sets with saturation counters smaller than the maximum value, which write back more frequently than those with maximum saturation counter values. However, SBC shares resources between two sets only when a set's saturation counter reaches its maximum. 
Writebacks may also be caused by cache flushes required for ensuring PCM crash consistency. In case cache flushes are required for the consistency of data in the persistent memory, to avoid causing consistency problems, the flushed dirty blocks need to be written back to the main memory. In other words, WALL cannot interfere with crash consistency flushes, and needs to treat normal writebacks and flush operations differently. The impact of flush operations on WALL depends on how often and when the flushes are required. With buffered epoch persistency [8], a set of write operations called an epoch are persisted together to allow write coalescing. In other words, data is persisted (i.e., or buffered to be persisted later) at specific points of the program execution specified by persist barriers. To implement epoch persistency, cache tags are extended with core and epoch ID fields to allow delaying or reordering of the writebacks. WALL can utilize the buffered epoch persistency model and still reduce the number of writebacks within the epochs. Using larger persistency epochs reduces the number of consistency writebacks; however, though, if a crash happens, then the program will need to be restarted from a relatively earlier state; so, there is basically a trade-off here for WALL. It is also worth noting that recent designs use non-invalidating flush operations (i.e., $c l w b$ instead of the cflush operations) to avoid the eviction of applications' working sets from the cache [12]. This enables WALL to continue keeping the blocks in the cache after persisting them in the main memory. Moreover, loose ordering consistency scheme proposed in Reference [17] relaxes the write ordering requirements with two mechanisms called eager commit and speculative persistence. So, such scheme can also be used with WALL, which basically delays the write back of some cache blocks to the main memory. Considering crash consistency when reducing the number of writebacks was not the focus of this study, and we leave it for the future work

\section{CONCLUSION}

In this article, a novel writeback-aware LLC management scheme is proposed to reduce the number of LLC writebacks to a PCM based main memory. We first investigate the non-uniformity of the LLC sets writebacks and based on that propose a writeback-aware set-balancing mechanism. To implement the set-balancing mechanism, we propose three novel partner assignment strategies, called contraction, expansion, and ConExp to pair sets with different behaviors more efficiently compared to the simple partner assignment strategy of WALL. In addition, we propose a simple but effective writeback-aware replacement policy to keep the frequently reused dirty lines of the sets in the cache. Our evaluation results show that our scheme can achieve a significant reduction in the total number of writebacks to PCM, thereby improving the system performance, energy efficiency, and PCM lifetime.

\section{ACKNOWLEDGMENTS}

Any opinions, findings, conclusions, or recommendations expressed in this article are those of the authors and do not necessarily reflect the views of the National Science Foundation.

\section{REFERENCES}

[1] M. Arjomand, M. T. Kandemir, A. Sivasubramaniam, and C. R. Das. 2016. Boosting access parallelism to PCM-based main memory. In Proceedings of the ISCA.

[2] M. V. Beigi, and G. Memik. 2016. TAPAS: Temperature-aware adaptive placement for 3D stacked hybrid caches. In Proceedings of the MEMSYS. 415-426.

[3] M. V. Beigi, and G. Memik. 2018. THOR: THermal-aware Optimizations for extending ReRAM lifetime. In Proceedings of the IPDPS.

[4] C. Bienia, S. Kumar, J. P. Singh, and K. Li. 2009. The PARSEC benchmark suite: Characterization and architectural implications. In Proceedings of the PACT. 72-81.

[5] CACTI-6.5. Retrieved from http: //hpl .hp.com: research /cacti/. 
[6] D. Chen, H. Jin, X. Liao, H. Liu, R. Guo, and D. Liu. 2017. MALRU: Miss-penalty aware LRU-based cache replacement for hybrid memory systems. In Proceedings of the DATE.

[7] Y. Choi, I. Song, M.-H. Park, H. Chung, S. Chang, and B. Cho. 2012. A 20nm 1.8v 8gb pram with 40mb/s program bandwidth. In Proceedings of the ISSCC. 46-48.

[8] J. Condit, E. B. Nightingale, C. Frost, E. Ipek, B. Lee, D. Burger, and D. Coetzee. 2009. Better i/o through byteaddressable, persistent memory. In Proceedings of the SOSP.

[9] X. Dong, C. Xu, Y. Xie, and N. P. Jouppi. 2012. Nvsim: A circuitlevel performance, energy, and area model for emerging nonvolatile memory. IEEE Trans. Comput.-Aided Design 31, 7 (2012), 994-1007.

[10] T. gem5-simulator. Retrieved from http://gem5.org/.

[11] IBM. A prototype phase-change memory cells. Retrieved from https://www.cnet.com/news/ibm-leaps-two-hurdlesfor-next-gen-memory/.

[12] A. Joshi, V. Nagarajan, M. Cintra, and S. Viglas. 2015. Efficient persist barriers for multicores. In Proceedings of the Micro.

[13] E. Kultursay, M. Kandemir, A. Sivasubramaniam, and O. Mutlu. 2013. Evaluating STT-RAM as an energy-efficient main memory alternative. In Proceedings of the ISPASS.

[14] B. C. Lee, E. Ipek, O. Mutlu, and D. Burger. 2009. Architecting phase change memory as a scalable DRAM alternative. In Proceedings of the ISCA. 2-13.

[15] H. H. S. Lee, G. S. Tyson, and M. K. Farrens. Eager writeback-a technique for improving bandwidth utilization. In Proceedings of the MICRO.

[16] K. Lim, P. Ranganathan, J. Chang, C. Patel, T. Mudge, and S. Reinhardt. 2008. Understanding and designing new server architectures for emerging warehouse-computing environments. In Proceedings of the ISCA. 315-326.

[17] Y. Lu, J. Shu, L. Sun, and O. Mutlu. 2014. Loose-ordering consistency for persistent memory. In Proceedings of the ICCD.

[18] NAS. The NAS parallel benchmarks. Retrieved from https://www.nas.nasa.gov/publications/npb.html.

[19] M. Poremba, T. Zhang, and Y. Xie. 2015. NVMain 2.0: Architectural simulator to model (non-)volatile memory systems. CAL 99 (2015), 1.

[20] B. Pourshirazi and Z. Zhu. 2016. Refree: A refresh-free hybrid DRAM/PCM main memory system. In Proceedings of the IPDPS.

[21] B. Pourshirazi and Z. Zhu. 2017. NEMO: An energy-efficient hybrid main memory system for mobile devices. In Proceedings of the MEMSYS.

[22] B. Pourshirazi, M. V. Beigi, Z. Zhu, and G. Memik. 2018. WALL: A writeback-aware LLC management for PCM-based main memory systems. In Proceedings of the DATE.

[23] M. K. Qureshi, V. Srinivasan, and J. A. Rivers. 2009. Scalable high performance main memory system using phasechange memory technology. In Proceedings of the ISCA. 24-33.

[24] M. K. Qureshi, M. M. Franceschini, and L. A. Lastras-Montaño. 2010. Improving read performance of phase change memories via write cancellation and write pausing. In Proceedings of the HPCA.

[25] M. K. Qureshi, M. M. Franceschini, A. Jagmohan, and L. A. Lastras. 2012. PreSET: Improving performance of phase change memories by exploiting asymmetry in write times. In Proceedings of the ISCA. 380-391.

[26] S. Raoux, G. W. Burr, M. J. Breitwisch, C. T. Rettner, Y. C. Chen, R. M. Shelby, M. Salinga, D. Krebs, S.-H. Chen, H. L. Lung, and C. H. Lam. 2008. Phase-change random access memory: A scalable technolog. IBM f. Res. Dev. 52, 4.5 (2008), 465-479.

[27] D. Rolán, B. B. Fraguela, and R. Doallo. 2009. Adaptive line placement with the set balancing cache. In Proceedings of the MICRO. 529-540.

[28] Z. Wang, S. Shan, T. Cao, J. Gu, Y. Xu, S. Mu, Y. Xie, and D. A. Jiménez. 2013. WADE: Writeback-aware dynamic cache management for NVM-based main memory system. Trans. Architect. Code Optim. 10, 4 (2013).

[29] Z. Wang, D. A. Jiménez, C. Xu, G. Sun, and Y. Xie. 2014. Adaptive placement and migration policy for an STT-RAMbased hybrid cache. In Proceedings of the HPCA.

[30] H. Wong, S. Raoux, S. B. Kim, J. Liang, J. P. Reifenberg, B. Rajendran, M. Asheghi, and K. E. Goodson. 2010. Phase change memory. Proc. IEEE 98, 12 (2010), 2201-2227.

[31] X. Wu, J. Li, L. Zhang, E. Speight, R. Rajamony, and Y. Xie. 2009. Hybrid cache architecture with disparate memory technologies. In Proceedings of the ISCA.

[32] F. Xia, D. Jiang, J. Xiong, M. Chen, L. Zhang, and N. Sun. 2014. DWC: Dynamic write consolidation for phase change memory systems. In Proceedings of the ICS. 211-220.

[33] F. Xia, D.-J. Jian, J. Xiong, and N.-H. Sun. 2015. A survey of phase change memory systems. f. Comput. Sci. Technol. 30, 1 (2015), 121-144.

[34] C. Xu, D. Niu, N. Muralimanohar, R. Balasubramonian, T. Zhang, S. Yu, and Y. Xie. 2015. Overcoming the challenges of crossbar resistive memory architectures. In Proceedings of the HPCA. 476-488. 
[35] D. Zhang, L. Ju, M. Zhao, X. Gao, and Z. Jia. 2016. Write-back aware shared last-level cache management for hybrid main memory. In Proceedings of the DAC.

[36] L. Zhang, B. Neely, D. Franklin, D. Strukov, Y. Xie, and F. T. Chong. 2016. Mellow writes: Extending lifetime in resistive memories through selective slow write backs. In Proceedings of the ISCA.

[37] M. Zhang, L. Zhang, L. Jiang, Z. Liu, and F. T. Chong. 2017. Balancing performance and lifetime of MLC PCM by using a region retention monitor. In Proceedings of the HPCA. 385-396.

[38] P. Zhou, B. Zhao, J. Yang, and Y. Zhang. 2014. Throughput enhancement for phase change memories. IEEE Trans. Comput 63, 8 (2014), 2080-2093.

Received July 2018; revised October 2018; accepted October 2018 